\title{
4
}

\section{AN APPLICATION OF EXTREME VALUE THEORY IN MODELING EXTREME SHARE RETURNS}

\author{
Konstantinos Tolikas \\ Cardiff University, UK
}

\begin{abstract}
Extreme value theory (EVT) methods are used to investigate the asymptotic distribution/s of the extreme minima and maxima of the Athens Stock Exchange daily returns over the period 1976-2001. Innovative aspects of this study include: (1) the generalized extreme value and generalized logistic distributions are considered, (2) Lmomentsratio diagrams are used to identify the distribution/s most likely to fit the extreme daily returns adequately, (3) the probability weighted moments method is used to estimate the parameters of the distribution/s, and (4) the Anderson-Darling goodness of fit test is employed to test the adequacy of fit. The generalized logistic distribution is found to provide adequate descriptions of the behavior of both the extreme minima and maxima over the period studied; however, the asymptotic distributions of extremes appear to become less fat tailed over time implying that the probability of a large daily return occurring is decreasing.
\end{abstract}

Keywords: Extreme value theory; L-moments; Anderson-Darling goodness of fit test; Generalized extreme value distribution; generalized logistic distribution.

\section{Introduction}

Longin (1996) defined the extreme return of an index to be the maximum or minimum return over a pre-specified time period; for example, the minimum daily return over a period of one week. These extremes can be the results of market corrections that can be characterized as usual market conditions or the results of political, economic, social, speculative or other unknown causes 
that can be characterized as market crises. Such large stock price movements can lead to enormous losses and consequently financial managers and regulators are increasingly interested in their associated probabilities. For example, the Bank for International Settlements (BIS, 1996) stipulated the use of value-at-risk $(\mathrm{VaR})^{\mathrm{a}}$ as a market risk measurement tool for financial institutions to calculate minimum capital requirements (MCR). This MCR aims to cover the potential losses that might accrue from a financial institution's market positions due to adverse market movements and should, therefore, help to avoid financial disasters. A usual assumption made in estimating $\mathrm{VaR}$ is that financial returns follow a normal distribution. Empirical evidence, however, suggests that most of the times, financial return distributions, especially those of high frequency (i.e., intraday or daily returns), deviate from normality (see, for example, Aparicio and Estrada (2001) for an assessment of the normality assumption of European stock returns). In this case the probabilities of extreme returns occurring will be underestimated and that can have a severe impact on investment strategies and on the stability of the financial system.

On the other hand, extreme value theory (EVT) is a special branch of statistics that focuses exclusively on these extremes and is increasingly used to model financial returns that exhibit non-normal behavior. The aim of this chapter is to examine the usefulness of innovative EVT methods by analyzing the asymptotic distribution of the extreme minima and maxima daily returns of a developing stock market; the Athens Stock Exchange (ASE). For that reason, daily prices of the ASE general index for the period 1976-1988 have been manually collected from the ASE Records Department and combined with daily prices for the period 1988-2001 collected from Datastream. This is a period of sufficient length to include major changes in the ASE as it has developed over the last decades. In addition, the EVT methods used in this chapter are quite innovative in financial analysis and their usefulness has not yet fully explored. Therefore, it is also aimed to introduce a new toolkit for those interested in the analysis of the extremal behavior of financial returns.

This chapter introduces a number of innovative points with respect to current applications of EVT in finance. First, in addition to the generalized

\footnotetext{
${ }^{\mathrm{a}} \mathrm{VaR}$ can be defined as the maximum loss of a portfolio over a particular time horizon and at a pre-specified confidence level (Jorion, 2000). Statistically, it can be defined as one of the lowest quantiles of the distribution of returns.
} 
extreme value (GEV) distribution, the generalized logistic (GL) is also considered as a model for the extremes of the ASE daily returns. Second, the distribution/s that are likely to best fit the empirical data are identified by using L-moments ratio diagrams and the parameters of these distributions are estimated by using the probability weighted moments (PWM) method. Finally, the Anderson-Darling (AD) test statistic is then used to test the goodness of fit of the chosen distribution/s to the empirical data.

This chapter is divided by the following sections. Section 2 provides a brief review of the literature regarding the EVT. The methodological steps are described in Sec. 3, while Sec. 4 describes the ASE general index daily returns. In Sec. 5, empirical results are obtained and the most likely distributions to fit adequately the extreme returns are identified. Finally, Sec. 6 concludes and summarizes the chapter.

\section{Extreme Value Theory}

Longin (1996) was one of the first to apply EVT in finance. In his seminal work he identified the distribution for the extreme daily returns of the S\&P500 over the period 1885-1990 to be the GEV. He also argued that EVT could be useful in VaR estimation, margin setting in future markets, and in regulating capital requirements for financial institutions. The GEV distribution was also found by McNeil (1999) to model adequately the S\&P500 annual minima during the period 1960-1987 who also illustrated that the drop of the S\&P500 index in October 1987 crash could have been predicted. Cotter (2001) applied EVT in estimating margin requirements in future markets and argued that EVT can lead to more realistic estimates. More recently Gettinby et al. (2004) investigated the distribution of the daily extreme returns in the UK stock market and found that the GL distribution provides accurate descriptions of both the minima and maxima extremes. Tolikas et al. (2007) also found that the GL distribution describes well the extremal behavior of daily returns in the German stock market and they also provided evidence that EVT can be useful in risk management since it can lead to more accurate VaR estimates. Important papers wherein the role of EVT in financial management is emphasized both theoretically and empirically, include Longin (2000), McNeil and Frey (2000) and Embrechts et al. (1998). A detailed description of the theory of extremes and its applications can be found in the books by Embrechts et al. (1997). 


\section{Methodology}

The EVT methods proposed in this study involve a number of steps. First, the frequency of financial returns should be chosen. This is influenced by the liquidity of the financial assets and since a general stock market index is used, daily logarithmic returns are employed. Second, the length of the selection interval over which the extremes are collected has to be specified. This choice directly affects the number of minima and maxima that are available for the analysis and should be sufficiently large to allow for the efficient estimation of the parameters of the extremes distribution. This is an arbitrary choice and in order to investigate both the behavior of parameters and the goodness of fit over successively increasing selection intervals; weekly, monthly, and quarterly extremes are considered. Without lost of generality, these extremes are collected over non-overlapping periods 5, 20, and 60 trading days, respectively. The behavior of the extremes distribution over time aggregation is also examined by dividing the series of weekly and monthly extremes into 2, 4, and 10 sub-periods and the quarterly extremes into 2 and 4 sub-periods.

The third step is to identify the distributions that are likely to model the empirical data adequately. The choice of an appropriate distribution may be affected by criteria such as its ability to reproduce the variability of the empirical data, sensitivity to data outliers, computational complexity, and the number of parameters to be estimated (Cunnane, 1985). Inspired by the work in the flood frequency analysis area, it was decided to focus on the GEV and GL distributions. Details of these distributions can be found in Appendix A. There are three parameter distributions that have been proved particularly effective in describing stochastic processes of extreme observations in environmental and engineering studies (see, for example, Peel et al., 2001). The parameters of these distributions are commonly referred as the location $(\beta)$, scale $(\alpha)$, and shape $(\kappa)$. The location and scale parameters are analogous to the mean and standard deviation, while the shape parameter is directly linked to the fatness of tails of the empirical distribution. Higher, in absolute terms, shape parameter values correspond to fatter tailed distributions and vice versa. Although the GEV has already been used to analyze financial extreme returns, the GL has only been used in a limited number of studies (Gettinby et al., 2004; Tolikas et al., 2007). This set of distributions includes as special cases stochastic processes such as, the normal, student- $t$, log-logistic and log-normal distributions, mixture of normal distributions or even ARCH processes (de Haan et al., 1989). 
The most likely distributions to fit the extremes are identified by using L-moments ratio diagrams. L-moments are linear combinations of ordered data and analogously to conventional moments they provide a summary statistic for probability distributions. ${ }^{\mathrm{b}}$ The main advantage of the L-moments is that being linear combinations of the ordered data they are more robust than the conventional moments in the presence of outliers ${ }^{\mathrm{c}}$ (Hosking, 1990; Royston, 1992; Sankarasubramanian and Srinivasan, 1999). They defined for any random variable $X$ which mean exists as follows (Hosking, 1990):

$$
\lambda_{r} \equiv r^{-1} \sum_{k=0}^{r-1}(-1)^{k}\left(\begin{array}{c}
r-1 \\
k
\end{array}\right) E X_{r-k: r}, \quad r=1,2, \ldots
$$

The first two L-moments, $\lambda_{1}$ and $\lambda_{2}$, can be regarded as measures of location and scale of a distribution. Additionally, the quantities $\tau_{r}=\lambda_{r} / \lambda_{2}$, where $r=3,4, \ldots$ are called the L-moment ratios of the random variable $X$ and the first two, $\tau_{3}$ and $\tau_{4}$, can be regarded as measures of skewness and kurtosis, respectively. The identification of the distributions that best fit the empirical data is implemented graphically by using a statistical distribution map, wherein the L-moment ratios equivalent to skewness and kurtosis are estimated from the data and plotted. ${ }^{\mathrm{d}}$ The same diagram contains the plots of the skewness and kurtosis of the theoretical distributions which are considered. The identification of the appropriate distribution for a particular data set is made, by choosing the distribution whose L-skewness and L-kurtosis curve passes closest to it. This allows the examination of the suitability of many distributions in just one diagram. For the purpose of distributions identification, the utility and the superiority of L-moment ratios diagrams against conventional moments diagrams is well illustrated in Peel et al. (2001) and Pandey et al. (2001).

The next step is to estimate the parameters of these most appropriate distributions. For that purpose the method of PWM is used. PWM are expectations of certain functions of a random variable $X$ with distribution function F, whose mean exists, and they first defined by Greenwood et al. (1979).

\footnotetext{
bThe book by Hosking and Wallis (1997) provides a detailed presentation of the L-moments theory.

${ }^{\mathrm{c}}$ This is because the calculation of conventional moments, like skewness and kurtosis, involve third and fourth powers; thus, greater weight is given to outliers.

${ }^{\mathrm{d}}$ Graphical methods have been used for a long time in social sciences to examine the goodness of fit of a theoretical distribution to the empirical data and have found to be particularly useful.
} 
A tractable definition is given by Hosking (1986).

$$
\alpha_{r}=E\left[X\{1-F(X)\}^{r}\right], \quad r=0,1, \ldots,
$$

where $E[X(\cdot)]$ is the expectation of the quantile function of a random variable $X$. The PWM method estimates a distribution's parameters by equating the sample moments to those of the fitted distribution. In the case of small samples this method is considered to be more efficient compared to the maximum likelihood (ML) method (Coles and Dixon, 1999). This is particularly important since, by definition, extremes are rare; even long observational periods can provide very few data points if, for example, annual minima are used. PWM are linearly related to L-moments by the following relationships (Hosking, 1986):

$$
\begin{aligned}
& \lambda_{1}=\alpha_{0}, \\
& \lambda_{2}=\alpha_{0}-2 \alpha_{1}, \\
& \lambda_{3}=\alpha_{0}-6 \alpha_{1}+6 \alpha_{2}, \\
& \lambda_{4}=\alpha_{0}-12 \alpha_{1}+30 \alpha_{2}-20 \alpha_{3},
\end{aligned}
$$

Hosking et al. (1985) showed that for the GEV distribution, estimates of parameters and quantiles made using the PWM method are estimated with at least $70 \%$ efficiency. They also showed that for shape parameter values in the range -0.5 to 0.5 and for samples of up to 100 observations, estimates generated by the PWM method have lower root-mean square error than estimates generated by the ML method. Additionally, Landwehr et al. (1979) found that for a special case of the GEV distribution, the Gumbel, the PWM method results in more efficient parameter estimates compared to the ML and conventional moments method.

Once the appropriate distribution/s have been identified and the parameters have been estimated it is essential to test the goodness of fit of the chosen distribution/s to the empirical data. Anderson and Darling (1954) defined a class of test statistics by:

$$
\int_{-\infty}^{\infty}\left[F_{n}(x)-F(x)\right]^{2} \phi(x) d F(x)
$$

where $F_{n}(x)$ is the empirical distribution function (EDF) of a random variable $X, F(x)$ is the cumulative distribution function (CDF) of $X, n$ is the number of observations, and $\phi(x)$ is a function that assigns weight to the 
squared differences $\left[F_{n}(x)-F(x)\right]^{2}$. When, $\phi(x)=\left[F_{n}(x)(1-F(x))\right]^{-1}$ the test statistic focuses on measuring discrepancies in both tails. ${ }^{\text {e }}$ Stephens (1976) and d'Agostino and Stephens (1986) have reported that the AD goodness of fit test to be more powerful than the Pearson chi-squared test for small sample sizes.

\section{Data Description}

The dataset used to illustrate the usefulness of EVT consists of 6466 daily logarithmic returns ${ }^{\mathrm{f}}$ of the ASE general index that cover the 26-year period of 2 January 1976 to 28 December 2001. The data for the period 1976-1988 have been manually collected from the ASE Records Department while the data for the period 1988-2001 has been obtained from Datastream. ${ }^{g}$ Prices for the second period take the account of any capital changes (e.g., dividend distributions, stock splits) but this is not the case for the prices of the first period. However, the focus is on the extreme returns which are collected over selection intervals and therefore, it can be reasonably assumed that any effect of capital changes is well diversified and no significant bias is present in the results.

Table 1 contains descriptive statistics of the dataset employed in the analysis. The ASE index has a daily mean return of $0.06 \%$ and a daily standard deviation of $1.64 \%$. The minimum daily return ${ }^{\mathrm{h}}$ is $-16.29 \%$ and occurred on $7 / 12 / 87$ while the maximum, $24.23 \%$, occurred two days after on 11/12/87. Furthermore, the daily returns distribution has a low value of skewness, 0.329 , but a rather high value of kurtosis, 19.045. The kurtosis

\footnotetext{
${ }^{\mathrm{e}}$ The $\mathrm{AD}$ test is a modification of the kolmogorov-Smirnoff goodness of fit test. However, the $\mathrm{AD}$ test depends on the particular distribution that is examined. This makes the AD a more effective test but it has the drawback that critical values must be produced by simulation.

${ }^{\mathrm{f}}$ Daily logarithmic returns are calculated according to the formula, $X_{t}=100 \ln \left(P_{t} / P_{t-1}\right)$, where $P_{t}$ is the index closing price at day $t$.

${ }^{g}$ Therefore, the dataset covers a period that computers were not used to record stock prices during which ASE prompted by EU directives as well as technological advances went considerable transformation. The Datastream code is TOTMKGR and the index is composed of the most heavily traded shares that aim to cover the $70 \%-80 \%$ of the total market capitalization.

${ }^{\mathrm{h}}$ Although the ASE was closed around the period of the stock markets crash on 19/10/87, the negative sentiment of investors affected the Greek stock market which lost $13.51 \%$ on $26 / 10 / 87$, $10.24 \%$ on $4 / 11 / 87,12.77 \%$ on $26 / 11 / 87$, and eventually $16.29 \%$ on $7 / 12 / 87$.
} 


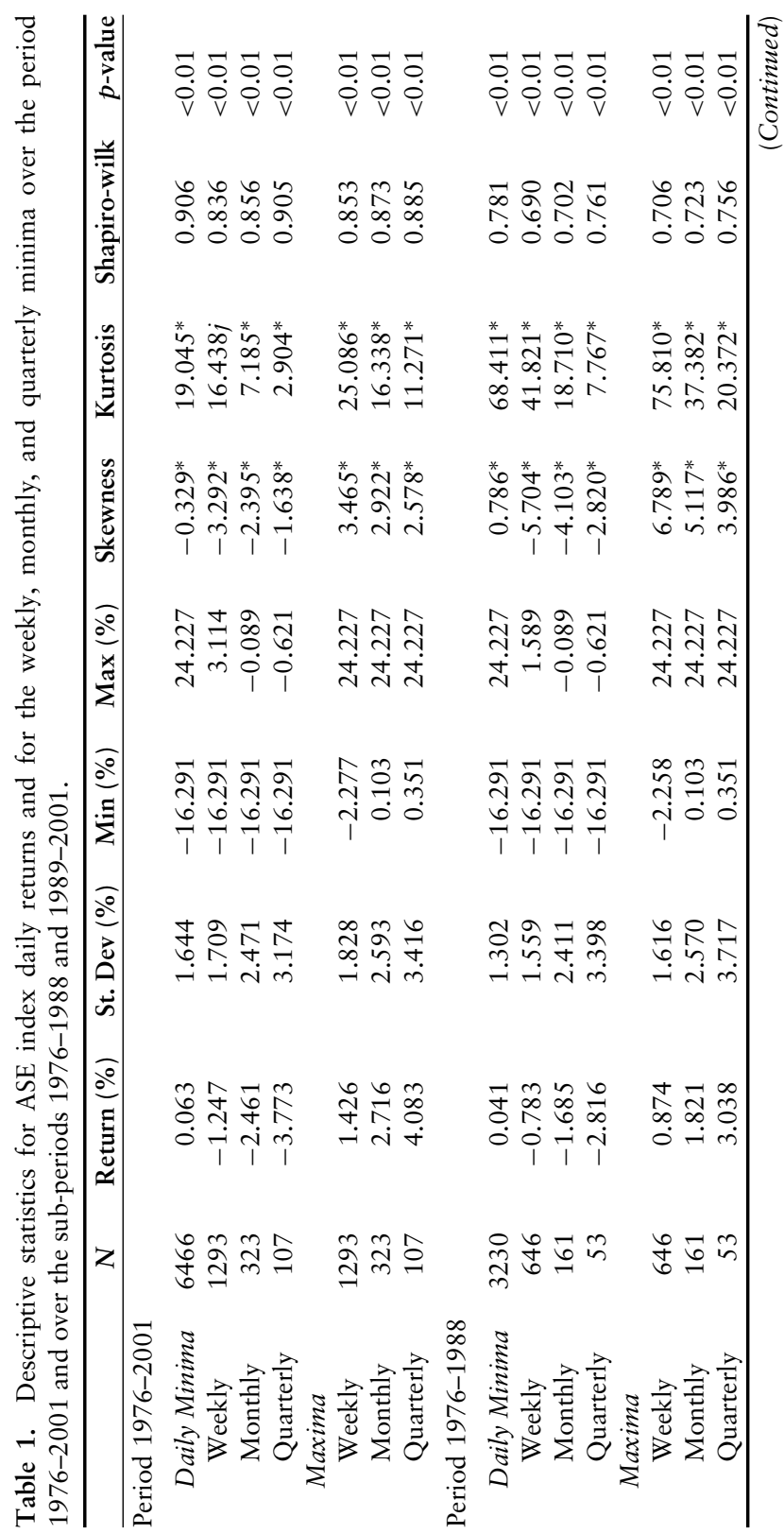




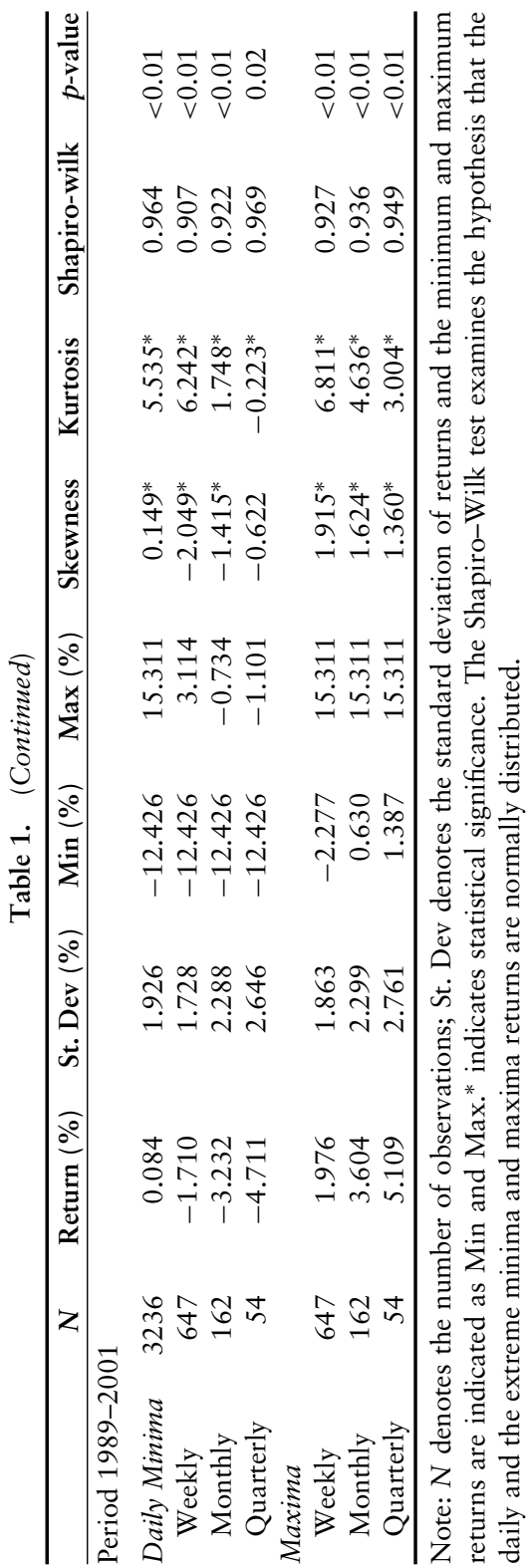


value indicate that the distribution of daily returns is fat tailed and therefore, it deviates from normality. ${ }^{i}$ This is also confirmed by the Shapiro-Wilk test statistic which rejects the normality assumption at the $99 \%$ confidence level. ${ }^{\mathrm{j}}$

In addition to the whole time period, descriptive statistics of the ASE daily returns for the sub-periods, 1976-1988 and 1989-2001 are also reported in Table 1. The mean daily return for the period 1976-1988 was $0.04 \%$ while that for the period 1989-2001 was double that figure, $0.08 \%$; however, standard deviation was also higher in the second period. Although skewness was low for both sub-periods, daily returns exhibited a large kurtosis of 68.41 in the first sub-period while in the second sub-period it was remarkably lower, 5.53. The normality hypothesis was also rejected for both sub-periods. A $Q-Q$ plot where quantiles of the daily returns empirical distributions for the two sub-periods are plotted against those of a normal distribution highlights the differences between the empirical distributions of the daily returns in the two sub-periods ${ }^{\mathrm{k}}$ (Fig. 1).

${ }^{\mathrm{i}}$ The values of skewness and kurtosis for the normal distribution are 0 and 3 , respectively.

iTo examine further the distribution of the daily returns, returns are standardized and the pairs of empirical percentiles $(1 \%, 99 \%),(5 \%, 95 \%)$, and $(10 \%, 90 \%)$ are compared with those of the standard normal distribution; $(-2.326,2.326),(-1.645,1.645)$, and $(-1.281,1.281)$, respectively. The comparison reveals that the pair of $(1 \%, 99 \%)$ empirical percentiles are too large, $(-3.102,3.096)$, while the pairs of $(5 \%, 95 \%)$, and $(10 \%, 90 \%)$ are too small, $(-1.315,1.490)$ and $(-0.842,0.933)$, respectively. Additionally, if the ASE daily returns were normally distributed then only 18 of the 6466 observations would lie outside plus or minus three standard deviations from the mean. This is because a well-known property of the normal distribution is that the $99.73 \%$ of the observations lie within plus or minus 3 standard deviations away from the mean. Therefore, only the $0.27 \%$ of total observations lies outside that range and since the normal distribution is symmetric, there should be a proportion of $0.135 \%$ in each tail. However, there are 144 observations that lie outside this range; 67 observations in the left tail and 77 observations in the right tail. Clearly, the empirical distribution of ASE daily returns exhibits more extreme observations than those implied by the normal distribution.

${ }^{\mathrm{k}}$ Furthermore, when the pairs of $(1 \%, 99 \%),(5 \%, 95 \%)$, and $(10 \%, 90 \%)$ empirical standardized percentiles are examined, it is revealed that for the period 1976-1988 all pairs are too small, $(-2.066,2.278),(-0.733,0.880)$, and $(-0.468,0.505)$, in comparison to those of the normal distribution; $(-2.326,2.326),(-1.645,1.645)$, and $(-1.281,1.281)$, respectively, while for the period $1989-2001$ the pairs of $(1 \%, 99 \%),(5 \%, 95 \%)$ are too large, $(-3.323,3.325)$ and $(-1.707,1.929)$ and the pair of $(10 \%, 90 \%)$ is too small, $(-1.167,1.280)$. Additionally, for the period 1976-1988, 59 observations lie outside the range of plus or minus three standard deviations, while only nine are expected, with 27 in the left and 32 in the right tail of the empirical distribution. For the period 1989-2001 there are 54 observations outside that range, while only nine are expected, with 25 in the left and 29 in the right tail of the empirical distribution. 


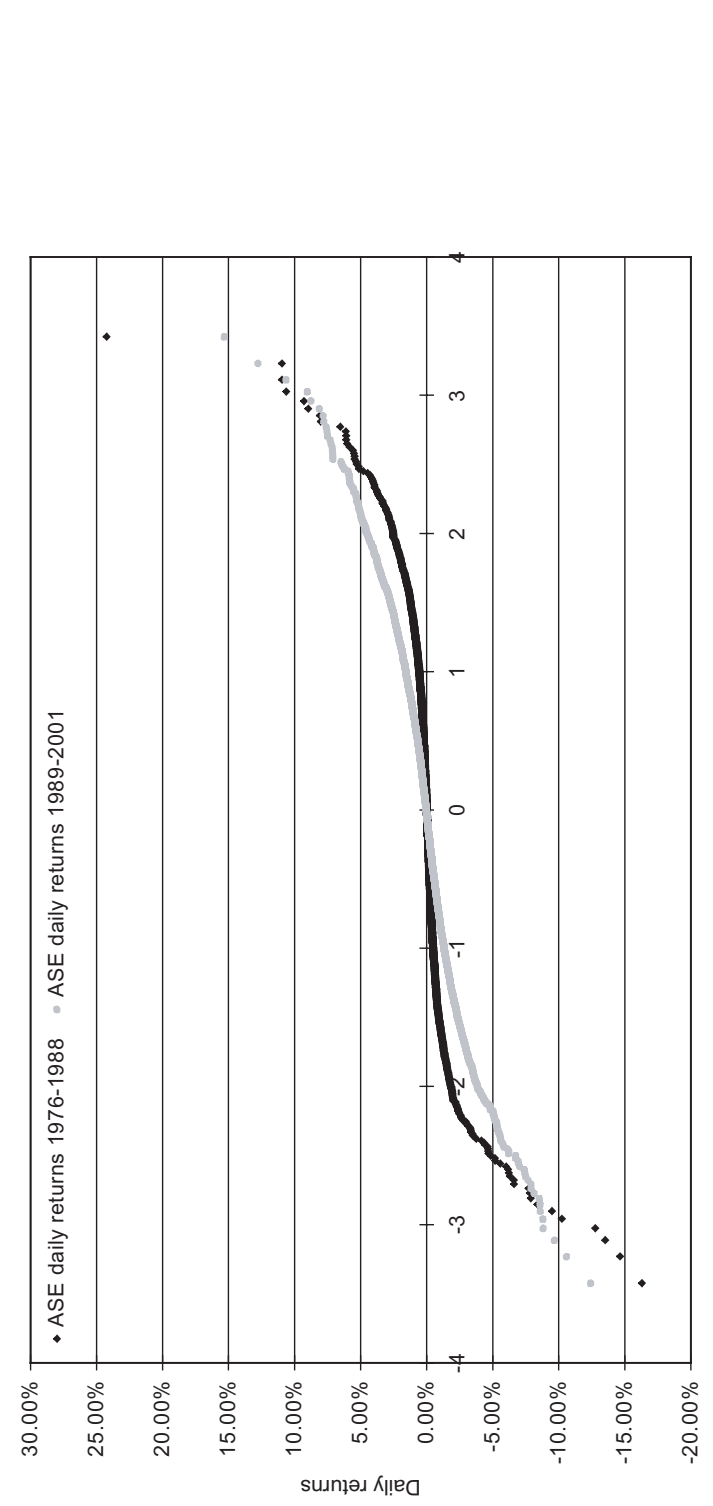

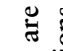

.

贾:

要要

氖

츨

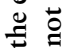

产

क

跣

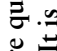

总

을

$\Xi$

훙

ว $\stackrel{2}{\frac{5}{\sigma}}$

N $\bar{\sigma}$

ह

象.

?

2

용

过

政

훙

o

일

O) हू

告

은

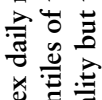

要

步

《.

可

Hin 


\section{Analysis of the Extreme Daily Returns}

\subsection{Identification of the appropriate distribution for the extreme daily returns}

Table 1 contains descriptive statistics for the weekly, monthly, and quarterly minima and maxima extreme daily returns which were collected over the 26-year period. The series of weekly and monthly extremes were also divided into 2, 4, and 10 sub-periods and the series of quarterly extremes into 2 and 4 sub-periods. The L-skewness $\left(\tau_{3}\right)$ and L-kurtosis $\left(\tau_{4}\right)$ were calculated and plotted for each of the sub-periods of the series of weekly, monthly, and quarterly extremes. The same diagram also contain the theoretical relationship between the L-skewness and L-kurtosis of different distributions. ${ }^{1}$

Figure 2 contains the plots of $\tau_{3}$ and $\tau_{4}$ for the 10 sub-periods of the weekly minima over the period 1976-2001. It can be noticed that the L-moment ratio points are mainly dispersed around the theoretical curves of the GL and the GEV distributions with most points lying above the GL curve. This is an interesting observation since higher L-kurtosis values correspond to fatter tailed distributions. Similar patterns appeared in the L-moment ratio diagrams for different sub-periods of the weekly, monthly, and quarterly minima. ${ }^{\mathrm{m}}$ The same graphical analysis was implemented for the extreme weekly, monthly, and quarterly maxima. Again, the L-moment ratio points are mainly dispersed around the theoretical curves of the GL and GEV distributions. Overall, although the L-moment ratio diagrams do not provide additional support in favor of either the GL or the GEV distribution the visual evidence suggests that the analysis should focus only on these two distributions.

\subsection{Parameter estimation and goodness of fit test}

The whole series of the weekly minima as well as different sub-periods of the weekly minima were fitted by the GL and GEV distributions using the PWM method. Table 2 contains the parameter estimates and the AD goodness of fit test for the 10 sub-periods. It is noticeable that both the GEV and GL did not provide an adequate fit to the whole data series as well as

\footnotetext{
${ }^{1} \mathrm{~A}$ single point represents two parameter distributions (e.g., the normal), whereas a three parameter distribution is represented by a curve (e.g., the GEV). The lower bound of all the distributions is also plotted.

${ }^{\mathrm{m}}$ These diagrams are not included in this chapter but they are available from the author upon request.
} 

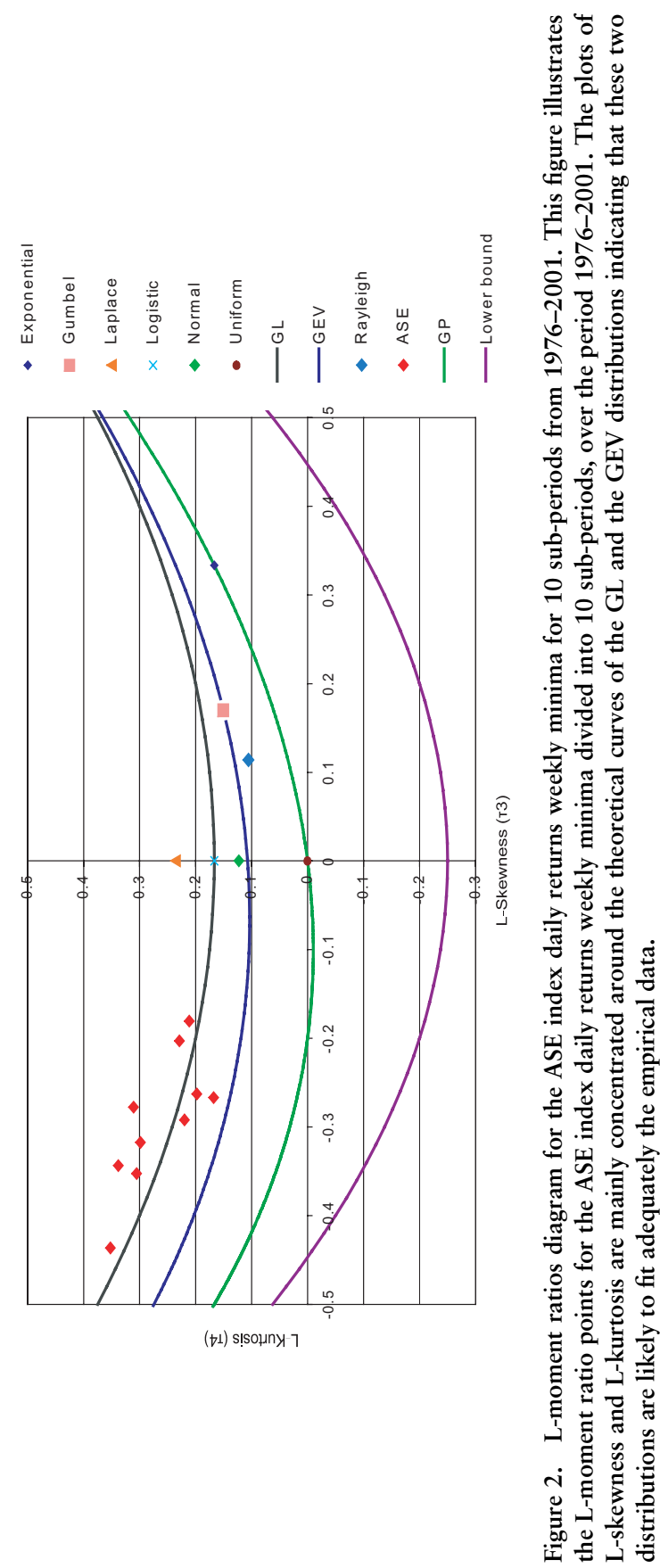


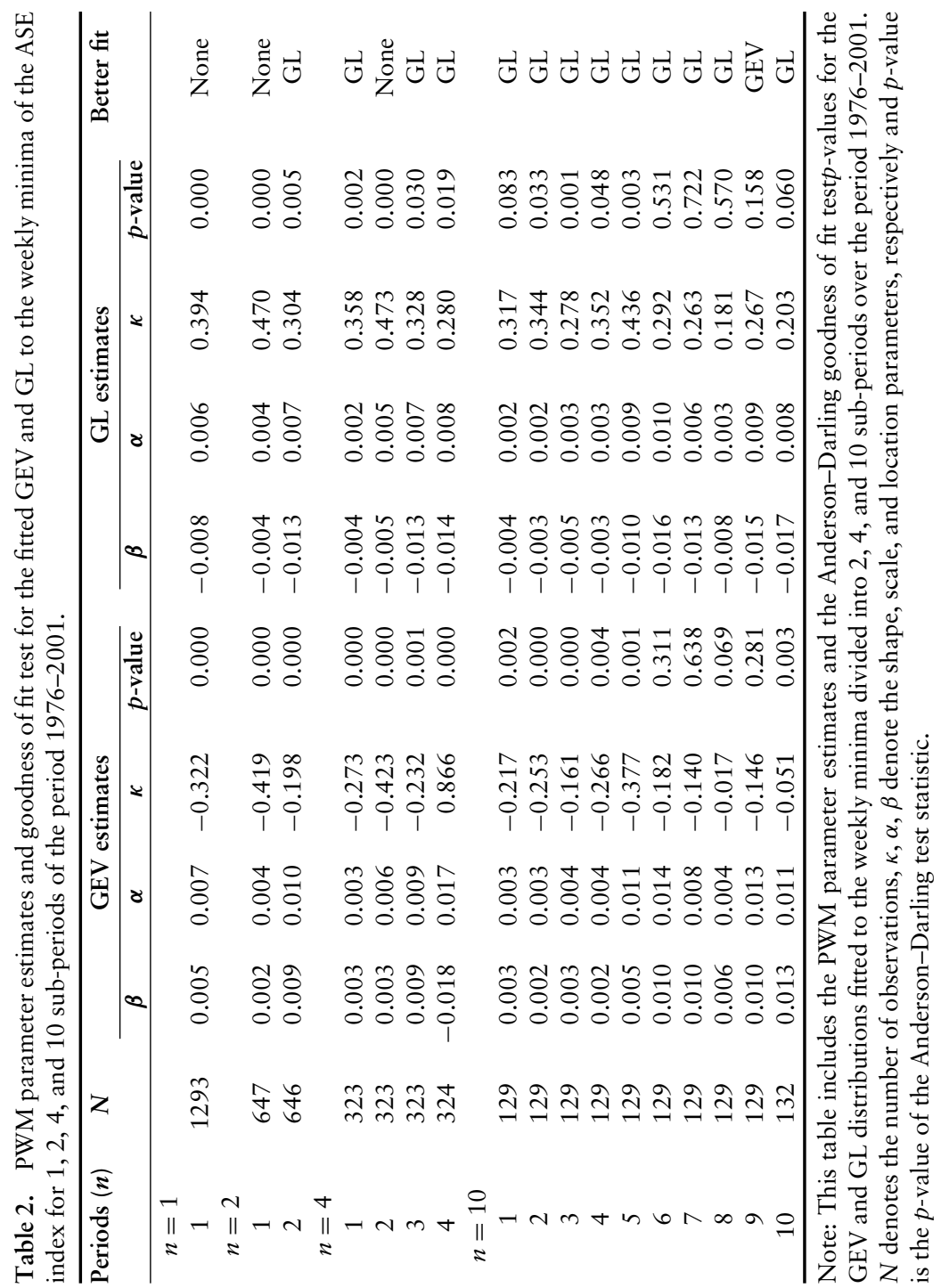


when 2 and 4 sub-periods were used. This is probably because the nature of extremes distribution is changing over time and therefore, when large timeperiods are used that encompass most of these changes the fitting becomes difficult. However, when 10 sub-periods were used the GL distribution fitted the empirical data adequately in six of the 10 sub-periods while the GEV in four of them. In comparison to the GEV, the GL fitted better the data in nine of the 10 sub-periods. However, a point that should be added in favor of the GEV distribution is that it performed significantly better in the last subperiods. This is probably because the distribution of extremes in the second half of the whole period is less fat tailed than it is in the first half. Therefore, the GEV, which is less fat tailed than the GL, improves its ability to fit the empirical data.

In Fig. 3 one could notice that the estimated scale and location parameters of the GL distribution over time behave in a stable fashion for the first periods but become volatile for the next sub-periods. This is because the second half of the total period contains larger negative daily returns than the first half. ${ }^{n}$ These large negative values probably reflect the effects of political uncertainty on the ASE during that period as well as the global volatile environment. In contrast, the shape parameter is more volatile in the first half than it is in the second half of the total period.

For the GEV distribution the parameters, in general, follow similar patterns (Fig. 4). The scale and location parameters appear to be stable for almost all periods that represent the first half of the 26-year period. In addition, the shape parameter estimates for the GEV vary more than the estimates for the GL and this is especially the case for the first half of the total period. Overall, even though both the GL and the GEV distributions fail to fit the whole sample of weekly minima, the GL performed better than the GEV when subperiods were used. Additionally, the parameter behavior over time appeared to be more stable for the GL than the GEV distribution.

The weekly maxima of the whole interval as well as of 2,4 , and 10 subperiods were also fitted by the GL and GEV distributions. ${ }^{\circ}$ Similarly, in the case of weekly minima both distributions failed to fit adequately the empirical data in the case of 1,2 , and 4 sub-periods. However, when the data was divided into 10 sub-periods both distributions improved their performance. In particular the GL fitted adequately in 7 of the sub-periods while the GEV

${ }^{\mathrm{n}}$ For example, the second half of the whole period contains the large drops of the ASE index with values of $-14.63 \%$ (3/10/88), $-12.42 \%$ (16/10/89), and $-10.59 \%$ (6/11/89), respectively. ${ }^{\circ}$ In the interest of brevity, these results are not included in the chapter. 


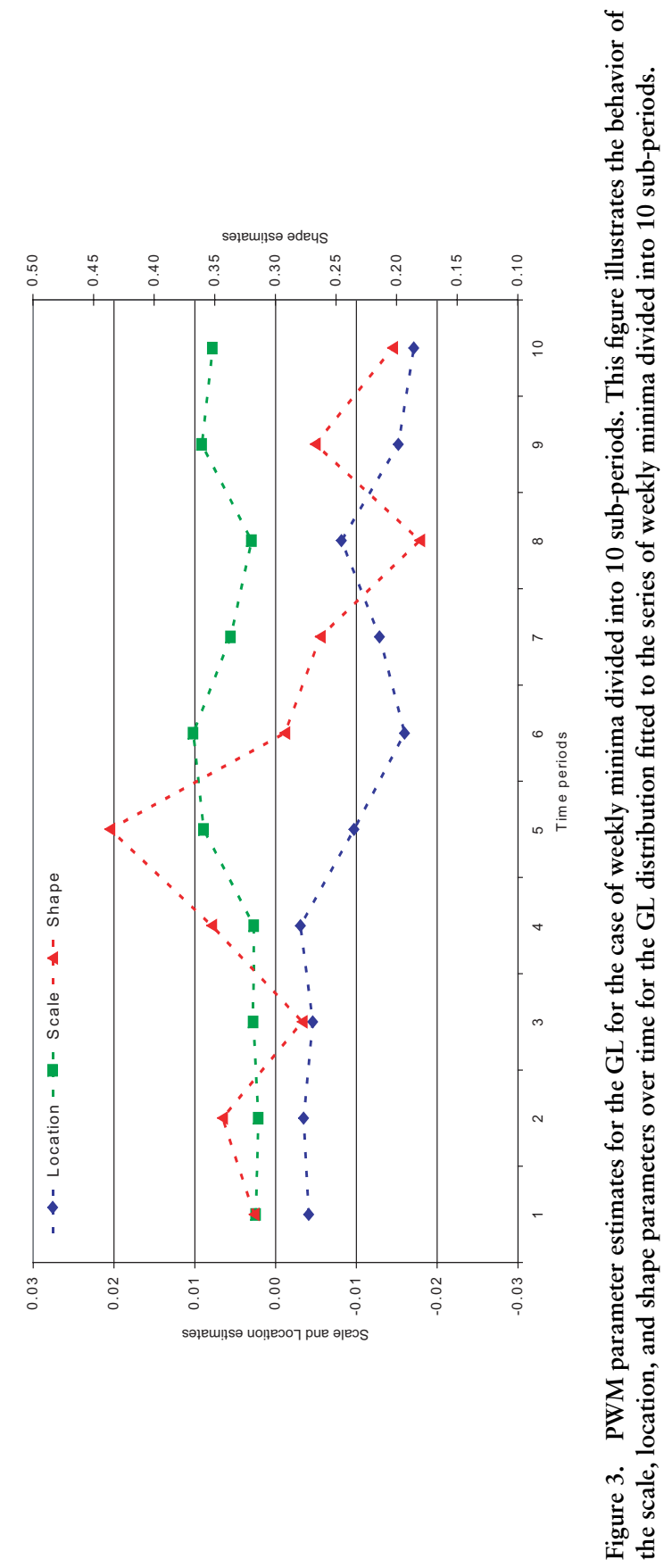




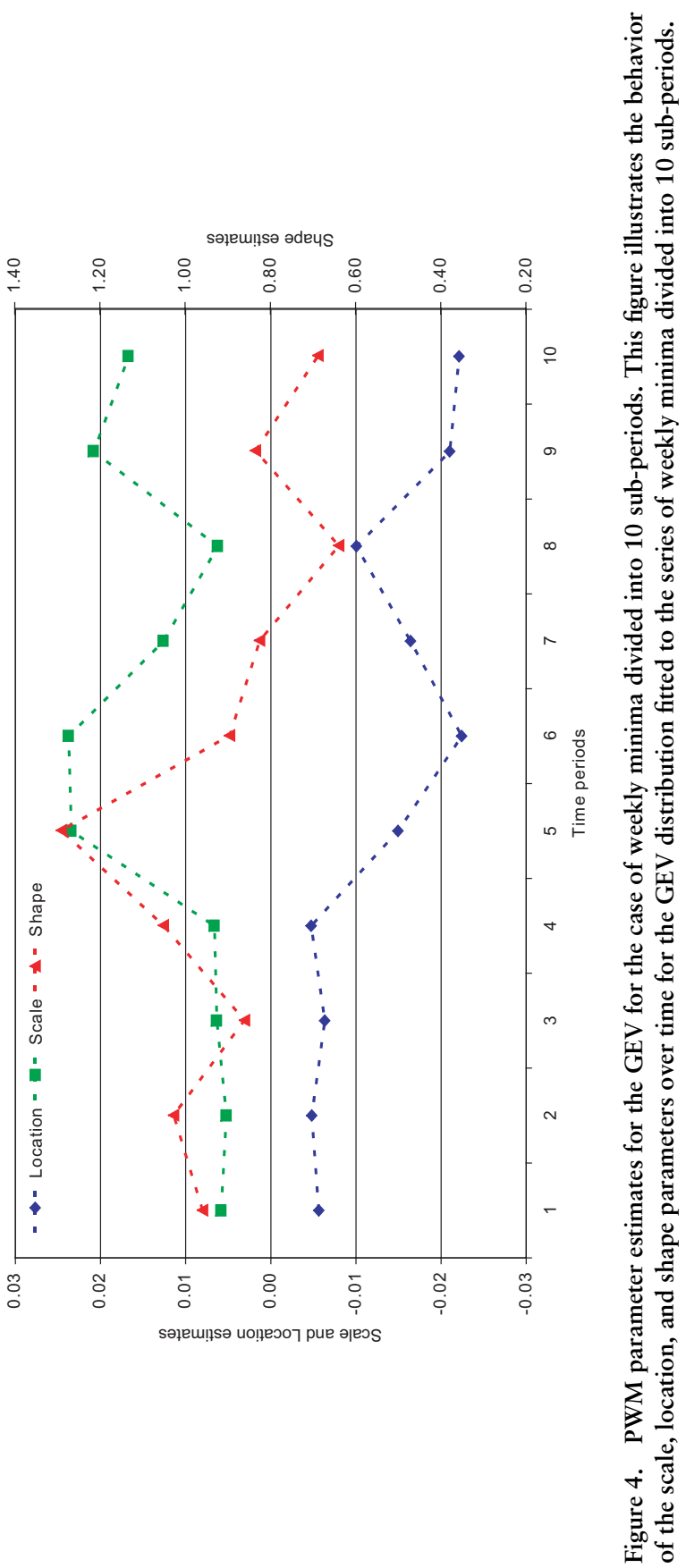


in the last 5 . It is noticeable that these 5 sub-periods refer to the period after 1988. An explanation is that the distribution of the ASE daily returns became less fat tailed in the second half of the period examined, thus the GEV improved its ability to fit adequately extreme daily returns. In terms of parameters' behavior over time, the patterns seem to be similar to those found in the case of weekly minima. For both the GL and GEV distributions, the location and scale parameters behave stable in the first half of the whole interval and they become volatile in the second half. Additionally, the shape parameter tends to take larger values, in absolute terms, in the first half rather than the second. Since larger shape parameter values in absolute terms indicate fatter tails it could be argued that the empirical distribution of the ASE index weekly maxima becomes less fat tailed over time. This finding can be interpreted as implying that the probability of a large extreme maxima occurring has decreased after 1988 (Table 3).

Overall both the GL and the GEV distributions appear to be able to provide an adequate fit to the empirical data. However, the GL seems to be the most appropriate distribution for the ASE extreme daily returns since its ability to fit the empirical data adequately is more consistent over time.

\subsection{GL and GEV parameter estimates behavior over time aggregation}

The monthly and quarterly minima and maxima of the ASE index were also fitted by the GL and GEV distributions. The reason for doing that is if a distribution fits adequately the extremes then its behavior should be consistent over different selection intervals of extremes. Results for the case of the monthly minima are presented in Table 4 and for the case of quarterly minima in Table 5. One could notice that none of the distributions fitted adequately the monthly minima for the whole period. In the case of 2 sub-periods the GEV fitted adequately in the first sub-period and the GL in none, while in the case of 4 sub-periods the GL fitted the data adequately in two while the GEV only in one sub-period. However, when the data was divided into 10 sub-periods both distributions performed better. The GEV fitted adequately in 6 and the GL in 7 sub-periods. In comparison, the GL fitted the data better than the GEV in 8 of the sub-periods. When quarterly minima were fitted by the GL and GEV both distributions failed to fit adequately the whole dataset while in the case of two sub-periods both the GEV and GL fitted adequately the data in the first sub-period with the GL giving a higher AD goodness of fit test $p$-value. Finally, in the case of four sub-periods the GL performed 


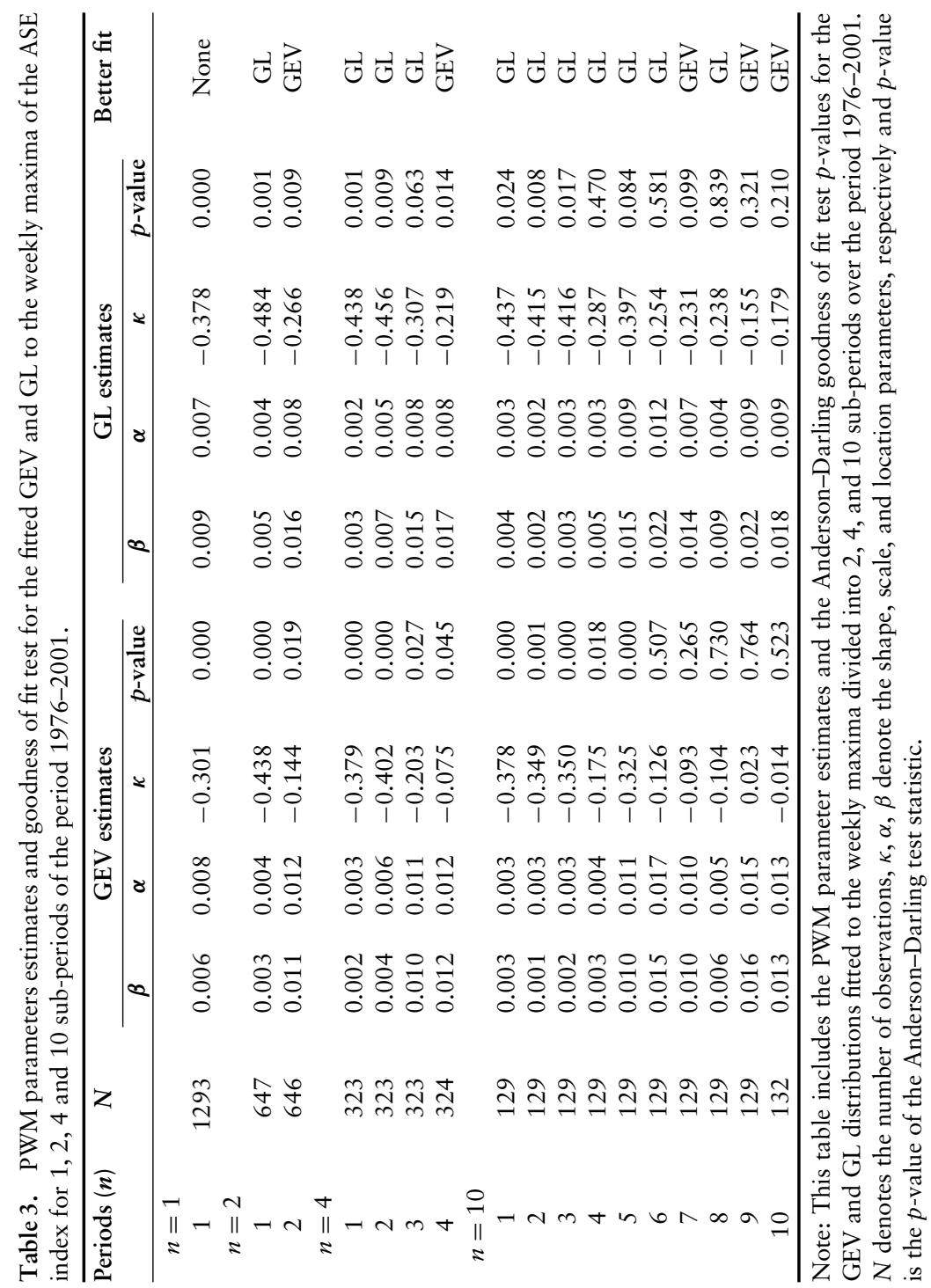




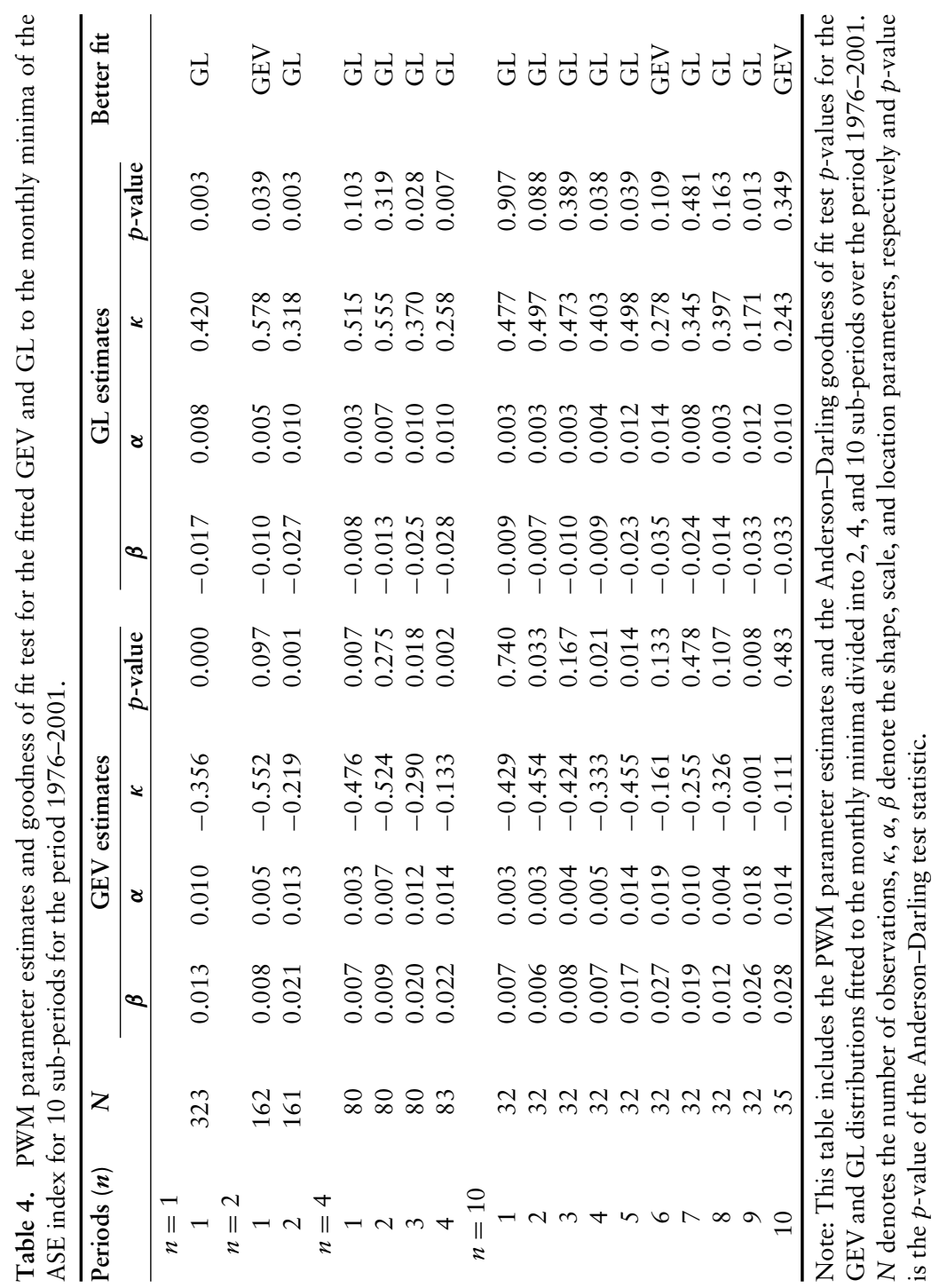




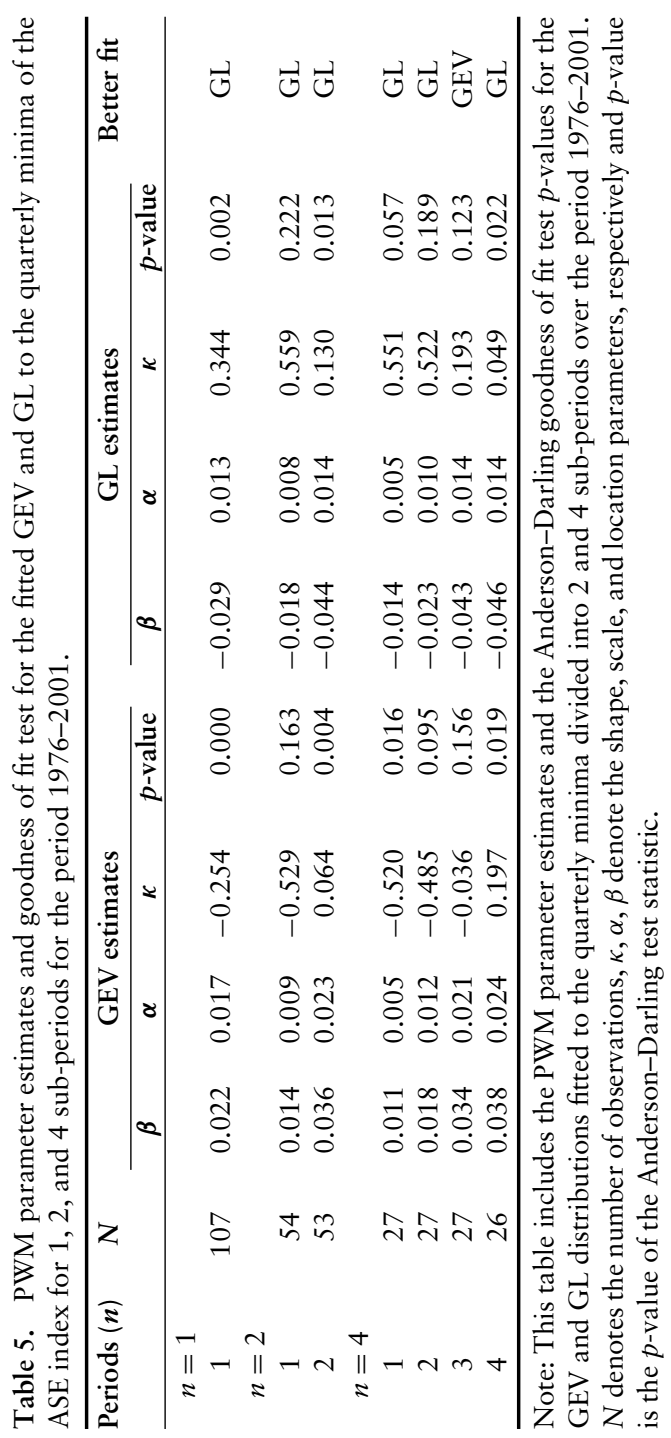




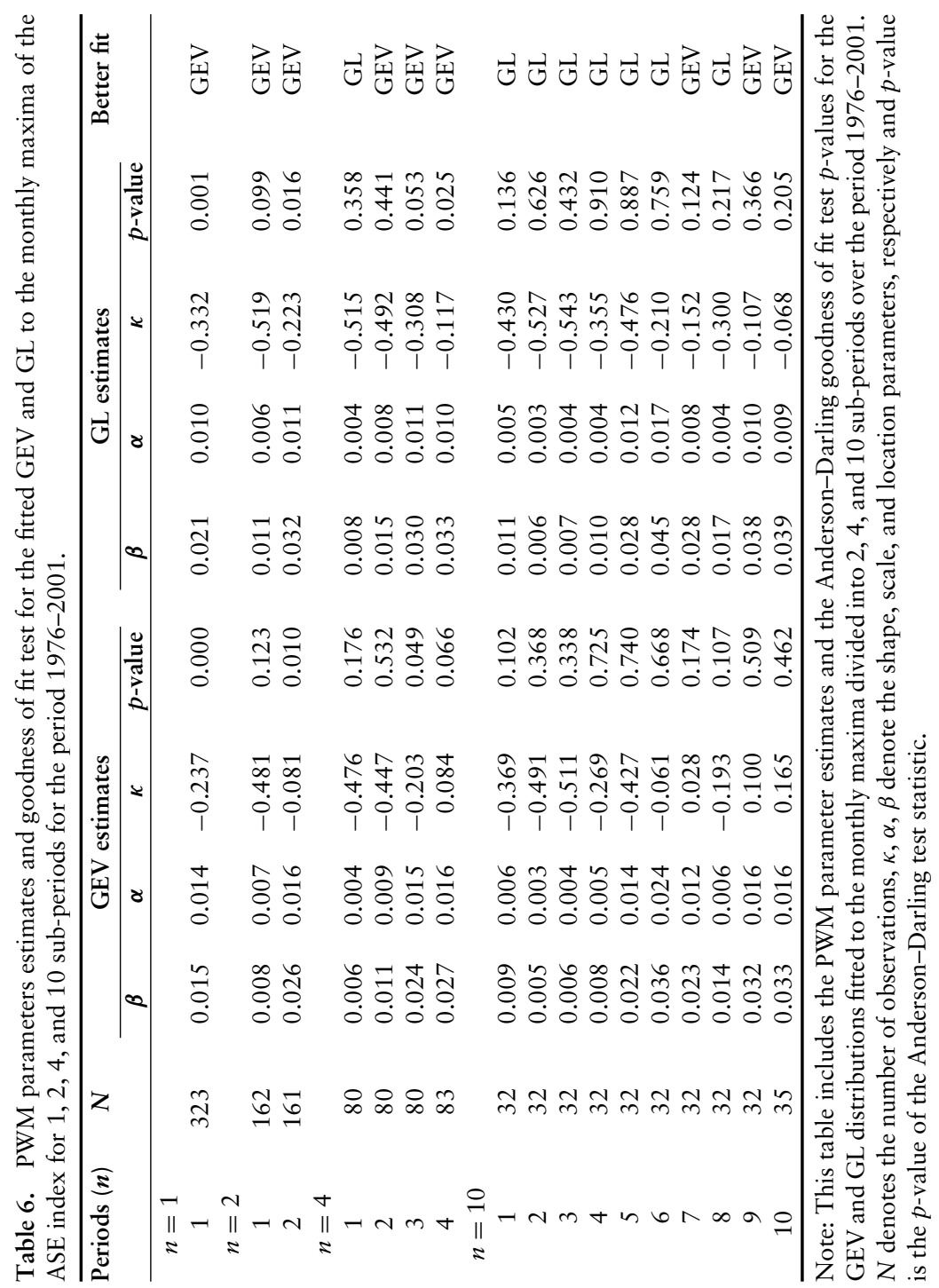




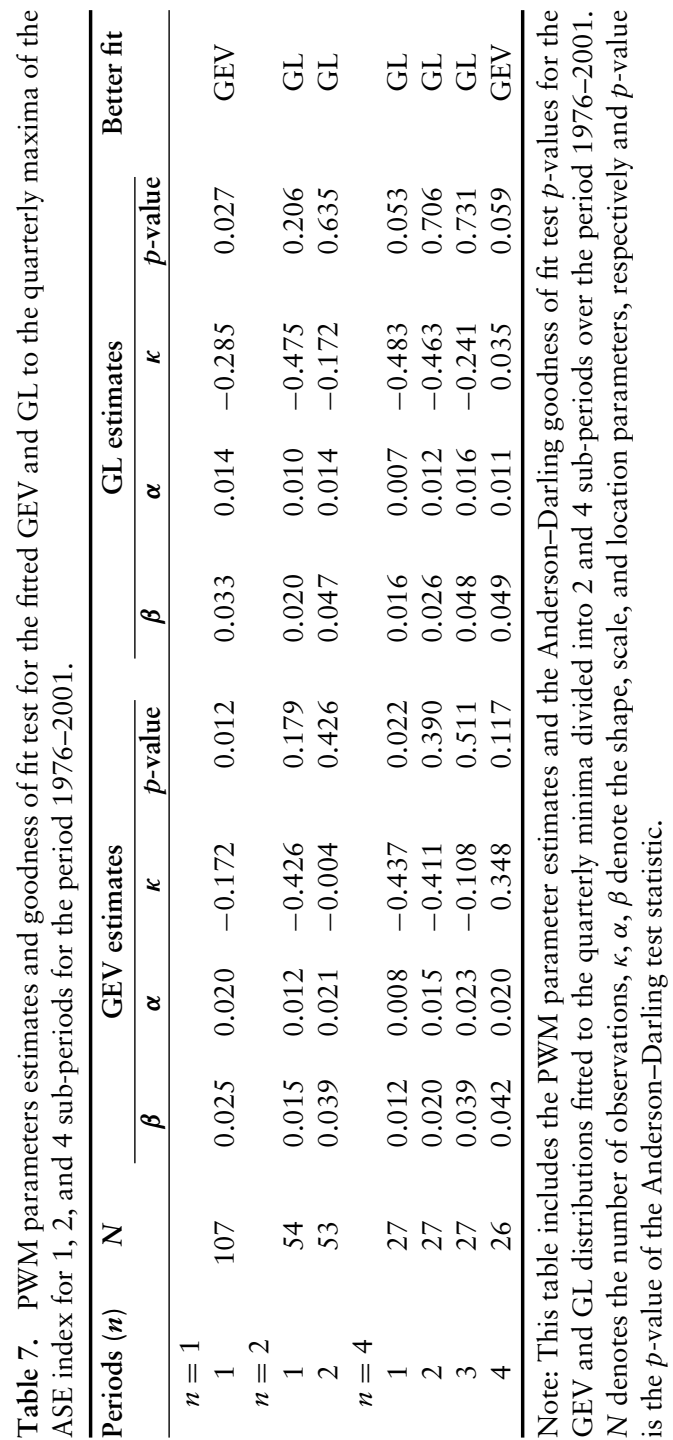


better than the GEV in 3 of them. Overall, it is the GL that describes consistently better the empirical data over different sub-periods and selection intervals.

The monthly and quarterly maxima of the ASE index were also been fitted by the GEV and GL distributions and the results are presented in Tables 6 and 7 , respectively. Similarly to the case of monthly and quarterly minima the results provided further support to the ability of the GL distribution to fit adequately the extreme daily share returns in the ASE. For example, when monthly maxima divided into 10 sub-periods were fitted the GL fitted 7 subperiods better than the GEV; although both distributions fitted adequately the data in all sub-periods. The same pattern appeared in the case of quarterly maxima divided into 4 sub-periods with the GL fitting better than the GEV in 3 sub-periods. In addition, the parameters appeared to behave in a similar fashion as in the case of the minima. The scale and location were more volatile during the second half of the whole period while the shape parameter exhibited a tendency to decrease over time from large to small values, in absolute terms, for both distributions. Overall, it is again found that the GL distribution provides a consistently better fit to the ASE index extreme maxima over the period 1976-2001.

\section{Conclusion}

This chapter employed innovative EVT methods in order to characterize the asymptotic distribution of the ASE extreme minima and maxima over the period 1976-2001. For this reason, prices from 1976 to 1988 were manually collected from the ASE and combined with prices from 1988 to 2001 collected from electronic sources. Weekly, monthly, and quarterly minima and maxima were then collected as the minimum and maximum daily returns over non-overlapping periods of certain length. A set of probability distributions comprising the GEV and GL was then considered, L-moment ration diagrams were used as an initial screening of the empirical data, the PWM method was used to estimate the parameters of the most likely distributions to fit the extremes and the AD goodness of fit test was used to assess the adequacy of fit. These EVT techniques can be useful to those interested in risk management since they comprise an additional toolbox for the analysis of extreme stock price movements and the estimation of the associated risk.

In terms of empirical results, it was found that the GL distribution provides adequate descriptions of the ASE index extreme minima and maxima 
for the period 1976-2001. This finding offers greater support to a limited number of studies (Gettinby et al., 2004; Tolikas et al., 2007) which have also found GL distribution to fit adequately financial extremes. Modeling adequately the tails of financial returns distributions can have important implications for risk management because this is where the really catastrophic events are located. The accurate estimation of their associated probabilities can therefore, lead to more accurate and helpful risk management tools. For example, research has shown that the use of EVT methods in VaR estimation can lead to more accurate estimates of the probabilities of big losses (see, for example, Tolikas et al., 2007; Longin, 2000).

Additionally, the behavior of the empirical distributions of both the minima and maxima extremes appeared to be time varying with a tendency to become less fat tailed over time. These results can be of particular importance to domestic and international investors as well as financial regulators, since less fat tailed distributions of extremes means that the probabilities of extremes occurring are decreasing and that could lead to increasing confidence amongst market participants and regulators. The latter can have practical implications for portfolio managers with respect to entering or exiting the Greek stock market.

\section{Appendix A}

The GEV and GL are three parameter distributions which have the following CDFs, quantile functions, and parameter estimates. The parameters $\kappa, \alpha$, and $\beta$ are called shape, scale, and location, respectively.

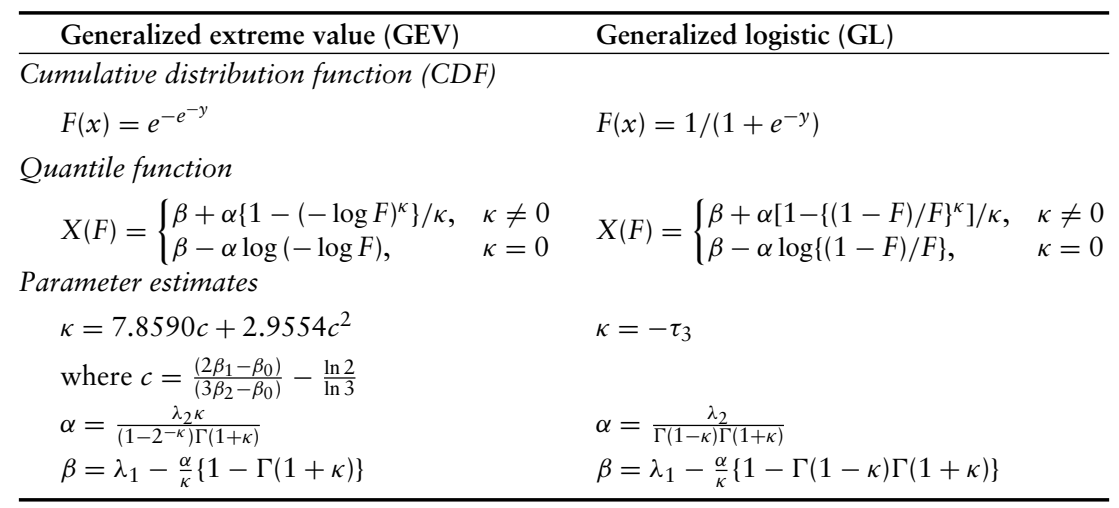




\section{Acknowledgment}

Financial support received from the Economic and Social Research Council (ESRC) is greatly acknowledged.

\section{References}

Anderson, TW and DA Darling (1954). A test for goodness of fit. The American Statistical Association, 49, 765-769.

Aparicio, FM and J Estrada (2001). Empirical distributions of stock returns: European securities markets, 1990-95. European Journal of Finance, 7(1), 1-21.

Basle Committee on Banking Supervision (1996). Amendment to the Capital Accord to Incorporate Market Risks, Basle: Bank for International Settlements.

Coles, S and MJ Dixon (1999). Likelihood-based inference for extreme value models. Extremes, 2(1), 5-23.

Cotter, J (2001). Margin exceedences for European stock index futures using extreme value theory. Journal of Banking and Finance, 25, 1475-1502.

Cunnane, C (1985). Factors effecting choice of distribution for flood series. Hydrologic Science Journal, 30(1), 23-36.

d' Agostino, RB and MA Stephens (1986). Goodness of Fit Techniques. New York: Marcel Dekker.

de Haan, L, IS Resnick, H Roótzen and CG de Vries (1989). Extremal behaviour of solutions to a stochastic difference equation with applications to $\mathrm{ARCH}$ process. Stochastic Processes and their Applications, 32, 213-224.

Embrechts, P, S Resnick and G Samorodnitsky (1998). Living on the edge. Risk, 11(1), 96-100.

Embrechts, P, C Kluppelberg and T Mikosh (1997). Modelling Extremal Events. Berlin: Springer-Verlag.

Gettinby, GD, CD Sinclair, DM Power and RA Brown (2004). An analysis of the distribution of extremes share returns in the UK from 1975 to 2000. Journal of Business Finance and Accounting, 31(5-6), 607-645.

Greenwood, JA, JM Landwehr, NC Matalas and JR Wallis (1979). Probability weighted moments: Definition and relation to parameters of several distributions expressable in inverse form. Water Resources Research, 15, 1049-1054.

Hosking, JRM and Wallis, JR (1997). Regional Frequency Analysis: An Approach Based on L-moments. Cambridge: Cambridge University Press.

Hosking, JRM (1990). L-moments: Analysis and estimation of distributions using linear combinations of order statistics. Journal of the Royal Statistical Society, Series B, 52, 105-124.

Hosking, JRM (1986). The theory of probability weighted moments. IBM TJ Watson Research Centre (\# 54860), pp. 1-160.

Hosking, JRM, JR Wallis and EF Wood (1985). Estimation of the generalised extreme-value distribution by the method of probability-weighted moments. Technometrics, 27(3), 251-261. 
Jorion, P (2000). Value-at-Risk: The New Benchmark for Controlling Market Risk. Chicago: McGraw-Hill.

Landwehr, JM, NC Matalas and JR Wallis (1979). Probability weighted moments compared with some traditional techniques in estimating Gumbel parameters and quantiles. Water Resources Research, 15, 1055-1064.

Longin, FM (2000). From value at risk to stress testing: The extreme value approach. Journal of Banking and Finance, 24, 1097-1130.

Longin, FM (1996). The asymptotic distribution of extreme stock market returns. Journal of Business, 69(3), 383-408.

McNeil, A and R Frey (2000). Estimation of tail-related risk measures for heteroscedastic financial time series: An extreme value approach. Journal of Empirical Finance, 7, 271-300.

McNeil, AJ (1999). Extreme Value Theory for Risk Managers. Internal Modelling and CAD II. London: Risk Books.

Pandey, MD, PHAJM Van Gelder and JK Vrijling (2001). Assessment of L-kurtosis criterion for quantile estimation. Journal of Hydrologic Engineering, 6(4), 284-292.

Peel, MC, QJ Wang, RM Vogel and TA McMahon (2001). The utility of L-moments ratio diagrams for selecting a regional probability distribution. Hydrological Sciences, 46(1), 147-155.

Royston, P (1992). Which measures of skewness and kurtosis are best? Statistics in Medicine, 11, 333-343.

Sankarasubramanian, A and K Srinivasan (1999). Investigation and comparison of sampling properties of L-moments and conventional moments. Journal of Hydrology, 218, 13-34.

Stephens, MA (1976). Asymptotic results for goodness-of-fit statistics with unknown parameters. Annals of Statistics, 4(2), 357-369.

Tolikas, K, A Koulakiotis and RA Brown (2007). Extreme risk and value-at-risk in the German stock market. To appear in European Journal of Finance. 
